\title{
Bone density and depression in premenopausal South African women: a pilot study
}

\author{
C Govender1, AME Du Plessis², P Bipath', D Povey3, G Viviers4', M Viljoen' \\ ${ }^{1}$ Department of Physiology, University of Pretoria, Pretoria, South Africa \\ 2Department of Psychiatry, University of Pretoria, Pretoria, South Africa \\ 3Department of Radiology, University of Pretoria, Pretoria, South Africa \\ ${ }^{4}$ Bone Density Unit, Medi-Clinic Heart Hospital, Pretoria, South Africa
}

\begin{abstract}
Objective: It is posited that the effect of depression on BMD is dependent on the severity of depression. Conflicting evidence exists regarding this possible association. This study investigated the association between depression and low bone mineral density (BMD). Methods: The hypothesis was investigated in a random sample of volunteers $(n=40)$ and in premenopausal female psychiatric patients $(n=5)$ diagnosed with recurrent severe major depression. The outcome measures were BMD (DEXA); depression (Beck Depression Inventory and Psychological General Well-being Scale) and 24-hour saliva cortisol levels (ELISA). In a comparison of women (4 of the 40 i.e. "control" subjects) with negligible symptoms of depression and the five patients with severe recurrent major depression- BMD, depression, saliva cortisol and bone turnover markers were measured and compared. Pro-inflammatory status (IL-1 and TNF-alpha) was investigated in the psychiatric patients only. Results: In the random - non clinical - sample of women ( $\mathrm{n}=40$ ), 26 exhibited normal BMD and 14 exhibited low BMD. Depressive symptoms and cortisol levels were not significantly different between these two groups. Women with severe recurrent major depression(n=5) exhibited lower median BMD T-scores, higher overall bone turnover and higher 24-hour cortisol levels compared to "control" subjects (n=4). The psychiatric patients also exhibited elevated IL- l levels. Conclusion: The effect of depression on BMD may be dependent on the depression severity. IL-1 and cortisol are possible mediators in depression-induced BMD loss.
\end{abstract}

Key words: Bone mineral density; Cortisol; Depression; Pro-inflammatory cytokines

Received: 04-08-2008

Accepted: 07-01-2010

\section{Introduction}

There is conflicting evidence regarding depression as a potential risk factor for osteoporosis. ${ }^{1}$ High cortisol and proinflammatory cytokine concentrations are frequently associated with depression ${ }^{2-4}$ and may cause bone resorption by suppressing type I collagen production, up-regulating osteoclastogenesis and decreasing the osteoblast population. ${ }^{5}$ Interleukins (IL) such as IL-1 and tumour necrosis factor alpha (TNF) have been shown to increase osteoclast maturation and increase resorption. 6,7

To explore the association between depression and bone mineral density (BMD), two study questions were examined:

Correspondence:

Ms Catherine Govender

Department of Physiology, University of Pretoria, PO Box 2034,

PRETORIA, 0001, South Africa

email: catherine.govender@up.ac.za
- Are there differences between depressive symptoms and cortisol levels in premenopausal women (non-psychiatric) with normal bone mineral density (BMD) vs. those women with low BMD?

- Do premenopausal women (psychiatric) suffering from severe recurrent major depression have normal BMD, bone turnover marker, cortisol and pro-inflammatory cytokine levels, compared to a non-psychiatric control group with negligible symptoms of depression?

\section{Method}

The non-psychiatric sample $(n=40)$ were recruited through two Gauteng hospitals and through advertising. The psychiatric sample $(n=5)$ comprised women with a single clinical diagnosis of recurrent, severe major depressive disorder who were approached through a local psychiatric clinic.

All subjects i.e. both psychiatric and non-psychiatric were females aged between 20 to 40 years. Excluded were subjects 
with any known psychological conditions other than depression, medical conditions including amenorrhoea or early menopause, history of use of anti-convulsants, calcitonin, thyroid hormones, alendronate, cyclosporin, anabolic steroids or bisphosphonates. Women suffering from depression with psychotic features or postpartum onset were excluded. Informed consent was obtained. The protocol was approved by the Ethics Committee of the University of Pretoria (90/2006).

Subjects were evaluated on total left femur, left femur head and lumbar BMD (measured on Dual-emission X-ray Absorptiometry or DEXA). The BMD readings were classified according to WHO criteria. ${ }^{8}$ Subjects with normal BMD on all three readings formed Group 1 ( $n=26)$; those with reduced BMD formed Group $2(n=14)$. The two groups were compared in terms of depressive symptoms [measured on the selfadministered Psychological General Well-Being Schedule (PGW) and Beck Depression Index (BDI)] and saliva cortisol (measured via ELISA using a solid phase enzyme immuno-assay from DRG Instruments). The PGW assesses psychological health within the past month of the subject's life. A score below 12 on the depressed mood subscale score reflects intense or frequent depressed mood. A score above 12 reflects never or rarely feeling depressed. ${ }^{9}$ The BDI measures the patient's self reported symptoms of depression as per the DSM-IV-TR. A score above 14 reflects depression. ${ }^{10}$

The patients recruited from the psychiatric clinic ( $\mathrm{n}=5$ ) were also investigated for BMD, features of depression and cortisol levels. In addition, bone turnover [osteocalcin, bone specific alkaline phosphatase (BSAP), type I collagen or pyridinoline cross-linked C-telopeptide (Pyd) and deoxypyridinoline (DPD) - evaluated by an external laboratory]; and pro-inflammatory status (IL-1 and TNF through ELISA) were also tested. Subjects from the non-psychiatric control group $(n=40)$ with a PGW score above 12 and a BDI score below 14 were approached to undergo the tests for bone turnover and inflammatory status to serve as controls for the psychiatric patients. Four subjects volunteered, constituting the "control" group.

Analyses were conducted using SAS $®$ and SPSS $®$ programmes. The level of significance was set at $\mathrm{P}<0.05$. Between-group differences for Groups 1 and 2 were analysed using Fisher's exact probability for categorical data and the MannWhitney test for continuous data. The statistical model suggested by Hruschka and colleagues was utilized to calculate cortisol levels over 24 hours for the two groups. ${ }^{11}$ This model did not require that all subjects provide the same number of readings. The following model describes the data model for cortisol readings collected at four points during a day: ${ }^{C O R T}{ }_{i j}=$ âo $+{ }^{\hat{a} l} \times$ TIME $+b_{i}+$ åj. The results for the psychiatric patients and control group are presented as median and standard deviations given the small group size.

\section{Results}

Groups 1 and 2 (non-psychiatric volunteers) differed significantly on all three mean BMD values ("p" range $=0.0001$ to 0.0008), contraception use $(p=0.002)$ and BMI $(p=0.032)$ only. Significant differences were not found on the depression measures. From the aforementioned cortisol model it was observed that the slopes were negative and similar for both groups (Group $1=-1.016$; SEM 0.659; Group $2=-1.266$; SEM 0.378). The intercepts too were very similar (Group 1=-9.219; SEM 0.264; Group 2=-10.432; SEM 0.968). Therefore similar readings were expected for both groups for waking cortisol and for the diurnal rate of change

Comparing the psychiatric patients $(\mathrm{n}=5)$ and their nonpsychiatric controls ( $\mathrm{n}=4$ ): the median ages, BMI, alcohol intake, contraception use and physical activity levels were similar. The median BMD T-scores were within normal limits for both groups (T-score $>-1$ ). The results for the psychiatric patients and controls on continuous measures are summarised in Table I.

\begin{tabular}{|l|l|l|}
\hline \multicolumn{2}{|l|}{ Table I: A summary of the scores and relevant normative data for the } \\
psychiatric patients (N = 5) and controls (N = 4)
\end{tabular}


The following differences were noted between the psychiatric sample and the non-psychatric controls:

1. The psychiatric sample exhibited a higher median depression score on the BDI and a lower median score on the PGW, an expected outcome.

2. Controls exhibited slightly higher median DEXA results than the psychiatric patients.

3. The median bone turnover marker values for the psychiatric sample were higher than those of the controls for all parameters. This indicates a trend of higher bone remodelling (both formation and resorption), in depressed patients when compared with non-depressed subjects.

4. The psychiatric sample's median 24-hour cortisol level $(8.334 \mathrm{ng} / \mathrm{ml})$ was higher than that of the control group $(6.45 \mathrm{ng} / \mathrm{ml})$.

5. The psychiatric sample's median IL- 1 reading (14.669 $\mathrm{pg} / \mathrm{ml}$ ) was well above the normative value of 4.721 $\mathrm{pg} / \mathrm{ml}$, indicating abnormally high levels of this proinflammatory cytokine. However, the median TNF reading was within the normative range (1.333 pg/ml).

\section{Discussion}

This study examined the possible association between depression and BMD. No significant difference was found between women (non-psychiatric) with normal and low $\mathrm{BMD}$ in terms of depression and cortisol measures. However, only moderate depression levels were represented and exploration of samples with higher depression scores is warranted.

When a small group of premenopausal women known to be suffering from recurrent severe major depression was compared with control subjects from within the nonpsychiatric sample of volunteers, it was observed that the clinical sample of depressed subjects showed lower BMD, higher bone resorption and bone synthesis marker levels and higher cortisol concentrations. Futhermore, IL-1 $\beta$ was elevated in the psychiatric patient group, suggesting a possible role for IL- $1 \beta$ in BMD loss in women with major depression. The elevated IL-1 $\beta$, in the presence of normal TNF $\alpha$ levels is consistent with the findings of Simon, et al., who reported a generalised pro-inflammatory response in depressed patients, with the exception of the TNF $\alpha$ levels. ${ }^{12}$ Unfortunately, the very small sample forces the observation into the category of anecdotal evidence and a much larger sample is needed before the results are given any credence.

\section{Conclusion}

It appears that the effect of depression on BMD is reliant on the clinical severity of the depression. The full profile of low BMD and up-regulated bone turnover may only be apparent in patients with severe recurrent major depression where elevated ILl- $\beta$ and cortisol are present. These results must be cautiously interpreted given the small sample, but the role of cortisol and proinflammatory cytokines in depression-related loss of BMD definitely warrants further investigation on larger populations of patients with major depression. Longitudinal studies with time-series measurements, consideration of antidepressant use and the depression- subtype will help gain a better understanding of depression as a risk for osteoporosis. ${ }^{13}$

\section{Funding, support and acknowledgements}

Ms Govender wishes to thank the Ernst and Ethel Eriksen Trust and the Research Committee of the Faculty of Health Sciences at the University of Pretoria for their generous funding of the project. We thank Rina Owen and Liebie Louw for the statistical analysis. We acknowledge the roles of Rhuhanda Bron and the staff of the Bone Density Unit at the Pretoria Heart Hospital. We thank the management of Pretoria Academic Hospital, the Department of Physiology at the University of Pretoria and Pretoria Heart Hospital for permission to conduct the study.

\section{References}

1. Yirmiya R, Bab I. Major Depression Is a Risk Factor for Low Bone Mineral Density: A Meta-Analysis. Biol Psychiatry 2009;66:423432 .

2. Vreeburg SA, Hoogendijk Witte JG, van Pelt J, DeRijk RH, Verhagen JCM, et al. Major Depressive Disorder and Hypothalamic-Pituitary-Adrenal Axis Activity: Results From a Large Cohort Study. Arch Gen Psychiatry 2009; 66(6):61 7-626.

3. Marques-Deak AH, Neto FL, Dominguez WV, Solis AC, Kurcgant $D$, Sato F, et al. Cytokine profiles in women with different subtypes of major depressive disorder. J Psychiat Res 2007;41:152-159.

4. Walsh MC, Choi Y. Biology of the TRANCE axis. Cytokine Growth FR 2003;14:251-263.

5. Von Der Mark K. Structure, biosynthesis and gene regulation of collagens in cartilage and bone. In Seibel MJ, Robins SP, Bilezikian JP. Dynamics of bone and cartilage metabolism, USA: Academic Press; 2006. p 3-40.

6. Eskandari F, Martinez PE, Torvik S, Phillips TM, Sternberg EM, Mistry S, et al. Premenopausal, Osteoporosis Women, Alendronate, Depression (POWER) Study Group. Low bone mass in premenopausal women with depression. Arch of Intern Med 2007; 67(21):2329-2336.

7. David J. Osteoimmunology: A view from the bone. Adv Immunol 2007;95:149-155.

8. Woolf AD, Dixon AS. Osteoporosis: A clinical guide. UK: Martin Dunitz; 1998.

9. Taylor JE, Poston WSC, Haddock CK, Blackburn GL, Heber D, Heymsfield SB, et al. Psychometric characteristics of the General Well-Being Schedule (GWB) with African-American women. Qual Life Res 2003;12:31-39.

10. Beck AT, Steer, RA, Brown GK. Beck Depression Inventory 2nd Edition, USA: Harcourt Brace \& Company, 1996.

11. Hruschka DJ, Kohrt BA, Worthman CM. Estimating between- and within-individual variation in cortisol levels using multilevel models. Psychoneuroendocrionology 2004;30: 698-714.

12. Simon NM, McNamara K, Chow CW, Maser RS, Papkostas GI, Pollack $M H$, et al. A detailed examination of cytokine abnormalities in Major Depressive Disorder. Eur Neuropsychopharm 2008;18:203-233.

13. Diem SJ, Blackwell TL, Stone KL, Yaffe K, Haney EM, Bliziotes MM, et al. Use of antidepressants and rates of hip bone loss in older women: the study of osteoporotic fractures. Arch of Intern Med 2007;167(12):1240-1245. 